\title{
Probing the Universe after Cosmological Recombination through the Effect of Neutral Lithium on the Microwave Background Anisotropies
}

\section{Citation}

Loeb, Abraham. 2001. "Probing the Universe after Cosmological Recombination through the Effect of Neutral Lithium on the Microwave Background Anisotropies." The Astrophysical Journal 555 (1): L1-5. https://doi.org/10.1086/321730.

\section{Permanent link}

http://nrs.harvard.edu/urn-3:HUL.InstRepos:41417368

\section{Terms of Use}

This article was downloaded from Harvard University's DASH repository, and is made available under the terms and conditions applicable to Other Posted Material, as set forth at http:// nrs.harvard.edu/urn-3:HUL.InstRepos:dash.current.terms-of-use\#LAA

\section{Share Your Story}

The Harvard community has made this article openly available. Please share how this access benefits you. Submit a story. 


\title{
PROBING THE UNIVERSE AFTER COSMOLOGICAL RECOMBINATION THROUGH THE EFFECT OF NEUTRAL LITHIUM ON THE MICROWAVE BACKGROUND ANISOTROPIES
}

\author{
ABRAHAM LOEB \\ Department of Astronomy, Harvard University, MS 46, 60 Garden Street, Cambridge, MA 02138; aloeb@cfa.harvard.edu \\ Received 2001 March 30; accepted 2001 May 16; published 2001 June 18
}

\begin{abstract}
I show that neutral lithium couples strongly to the cosmic microwave background (CMB) through its $6708 \AA$ resonant transition after it recombines at $z \sim 500$. At observed wavelengths of $\leqslant 335 \mu \mathrm{m}$, the CMB anisotropies are significantly altered since the optical depth for resonant scattering by neutral lithium is substantial, $\tau_{\mathrm{Li}} \sim$ 0.5 . The scattering would suppress the original anisotropies but will generate strong new anisotropies in the CMB temperature and polarization on subdegree scales $(l \gtrsim 100)$. Observations at different wavelengths in this spectral regime can probe different thin slices of the early universe. The anisotropy noise contributed by continuum radiation from foreground far-infrared sources could be taken out by subtracting maps at slightly different wavelengths. Detection of the above effects can be used to study structure at $z \lesssim 500$ and to constrain the primordial abundance and recombination history of lithium.
\end{abstract}

Subject headings: cosmic microwave background - cosmology: theory

\section{INTRODUCTION}

The dynamical coupling between the baryons and the cosmic microwave background (CMB) during the epoch of cosmological recombination $\left(z \sim 10^{3}\right)$ is commonly assumed to be mediated only through Thomson scattering (Peebles \& Yu 1970). This coupling becomes ineffective when hydrogen recombines, at around the same time when the universe becomes transparent, and hence it leaves a clear signature on the temperature anisotropies of the CMB (White, Scott, \& Silk 1994 and references therein). Forthcoming satellite missions, such as $M A P^{1}$ in 2001 or Planck ${ }^{2}$ in 2007, offer the sensitivity to probe deviations from this simple model at the percent level (Scott 2000; Yu, Spergel, \& Ostriker 2001).

In this Letter, I examine the potential significance of the coupling between the cosmic gas and the CMB due to resonant line transitions of neutral atoms. For such transitions, the integral of the absorption cross section over frequency,

$$
\int \sigma(\nu) d \nu=f_{12} \frac{\pi e^{2}}{m_{e} c},
$$

is typically many orders of magnitude larger than for Thomson scattering, where $f_{12}$ is the absorption oscillator strength (Rybicki \& Lightman 1979, p. 102). For example, the Ly $\alpha$ transition of hydrogen provides an average cross section that is 7 orders of magnitude larger than the Thomson value, when averaged over a frequency band as wide as the resonant frequency itself $[(\Delta \nu / \nu) \sim 1]$. Since the abundance of atoms in excited states is highly suppressed by large Boltzmann factors at the recombination redshift, we consider only transitions from the ground state. For helium, these transitions correspond to photon frequencies that are too far on the Wien tail of the CMB spectrum and only couple to a negligible fraction of the radiation energy density. Hence, we focus our discussion on hydrogen and lithium.

In $\S 2$, we derive the resonant line opacity and drag force for atoms that move relative to a blackbody radiation field. In $\S 3$, we consider the cosmological implications of these results for hydrogen and lithium. Finally, in $\S 4$, we summarize the

\footnotetext{
${ }^{1}$ See http://map.gsfc.nasa.gov.

${ }^{2}$ See http://astro.estec.esa.nl/Planck.
}

main conclusions of this work. Throughout this Letter, we adopt the density parameters $\Omega_{0} \approx 0.3$ for matter and $\Omega_{b}=0.04$ for baryons and a Hubble constant $H_{0} \approx 70 \mathrm{~km} \mathrm{~s}^{-1} \mathrm{Mpc}^{-1}$. The effect of the cosmological constant can be neglected at the redshifts of interest here.

\section{DRAG FORCE AND OPACITY DUE TO A RESONANT LINE}

Consider a single atom moving at a nonrelativistic peculiar velocity $\boldsymbol{v}$ relative to an isotropic blackbody radiation field of temperature $T$. Because of its motion, the atom sees a dipole anisotropy in the radiation temperature, $T_{a}(\theta)=T[1+(v / c) \times$ $\cos \theta]$, where $\theta$ is the angle relative to its direction of motion (Peebles 1993, p. 157). We assume that the atom couples to the radiation through a resonant line of frequency $\nu_{0}$ with $\left(h \nu_{0} / k T\right) \gg 1$. Hence, the line resonates in the Wien tail of the spectrum, for which the radiation energy density per unit frequency (in units of ergs $\mathrm{cm}^{-3} \mathrm{~Hz}^{-1}$ ) is given by

$$
\begin{aligned}
u_{\nu}(\theta)= & \frac{8 \pi h \nu^{3}}{c^{3}} \exp \left[-\frac{h \nu}{k T_{a}(\theta)}\right] \approx \frac{8 \pi h \nu^{3}}{c^{3}} \exp \left(-\frac{h \nu}{k T}\right) \\
& \times\left(1+\frac{h \nu}{k T} \frac{v}{c} \cos \theta\right),
\end{aligned}
$$

where we have used the fact that $[(h \nu / k T)(v / c) \cos \theta] \ll 1$. Absorption of a resonant photon by the atom is followed by isotropic emission in the atom's rest frame, and so the net drag force (momentum transfer per unit time) is given by

$$
F=-\int_{0}^{\infty} d \nu \frac{1}{2} \int_{-1}^{1} d \cos \theta \sigma(\nu) u_{\nu}(\theta) \cos \theta
$$

Since the absorption cross section is sharply peaked around $\nu_{0}$, we may use equation (1) to get

$$
\boldsymbol{F}=-\left[\frac{8 \pi^{2} h^{2} f_{12} \nu_{0}^{4}}{3 c^{3} k T} \frac{e^{2}}{m_{e} c^{2}} \exp \left(-\frac{h \nu_{0}}{k T}\right)\right] \boldsymbol{v} .
$$

For an ensemble of atoms of density $n_{a}$, which are all moving at the same peculiar velocity $\boldsymbol{v}$, the force per unit volume is 
given by $n_{a} \boldsymbol{F}$. If the resonant atoms are embedded within a hydrogen plasma of total (free + bound) proton density $n_{p}$ and are strongly coupled to the plasma through binary particle collisions, then the cosmological equation of motion for the gas as a whole reads

$$
\left(m_{p} n_{p}+m_{a} n_{a}\right)\left(\frac{d \boldsymbol{v}}{d t}+H \boldsymbol{v}\right)=n_{a} \boldsymbol{F}
$$

or, equivalently,

$$
\frac{d v}{d t}+H v=-\frac{v}{t_{a}}
$$

where $H=(\dot{a} / a)$ is the Hubble expansion rate and $t_{a}$ is the characteristic time over which the peculiar velocity of the gas is damped because of the drag force on the resonant atoms,

$$
t_{a}(t) \equiv \frac{3 m_{p} \lambda_{0}^{4}}{8 \pi^{2} h^{2} c f_{12}} \frac{m_{e} c^{2}}{e^{2}} \frac{k T}{X_{a}} \exp \left(\frac{h \nu_{0}}{k T}\right)
$$

Here $\lambda_{0}=c / \nu_{0}$ is the resonant wavelength, $m_{p}$ is the proton mass, and $X_{a} \equiv n_{a} /\left(n_{p}+A_{a} n_{a}\right)$, where $A_{a}=m_{a} / m_{p}$ is the atomic weight of the resonant atoms. In the cosmological context, $T=2.725 \mathrm{~K} \times(1+z)$ is the $\mathrm{CMB}$ temperature (Mather et al. 1999). Both $T$ and $X_{a}$ are functions of cosmic time, and so equation (6) admits the solution, $v=$ $\left[v_{0} / a(t)\right] \exp \left[-\int d t / t_{a}(t)\right]$, where $v_{0}=$ const and $a(t)$ is the cosmological scale factor. In general, the right-hand side of equation (6) should include other terms that source the peculiar velocity field, such as gravitational or pressure forces due to density inhomogeneities. The significance of the drag force can be calibrated in terms of the Hubble time, $H^{-1}(z)$, through the product $H t_{a}$. For the redshifts of interest, $H(z) \approx \sqrt{\Omega} H_{0}(1+z)^{3 / 2}$.

The assumption of an isotropic radiation background is only valid if the moving fluid element is optically thin to resonant scattering. In the opposite regime of high opacity, the background photons are isotropized locally through multiple scatterings and exert a reduced drag force on an atom embedded deep inside the moving fluid element. The Sobolev optical depth for resonant scatterings in an expanding and nearly uniform universe is given by (Sobolev 1946; Dell'Antonio \& Rybicki 1993)

$$
\tau_{a}(z)=f_{12} \frac{\pi e^{2}}{m_{e} c} \frac{\lambda_{0} n_{a}(z)}{H(z)}=\frac{A_{21}\left(g_{2} / g_{1}\right) \lambda_{0}^{3} n_{a}(z)}{8 \pi H(z)},
$$

where $A_{21}$ is the transition probability per unit time for spontaneous emission between the two energy levels (per second) and $\left(g_{2} / g_{1}\right)$ is the ratio between the statistical weights of the excited and ground states. The optical depth is dominated by the velocity gradient of the Hubble flow because gravitationally induced peculiar velocities perturb this flow only slightly at the early cosmic times of interest here.

For $\tau_{a} \gg 1$, the flux of resonant photons inside the moving fluid element is reduced by a factor of $\sim \tau_{a}$ (Rybicki \& Hummer 1978), and so the drag force in equation (3) is lowered by the same factor. This reduction has dramatic consequences for the resonant Ly $\alpha$ transition of neutral hydrogen, as we show next.

\section{IMPLICATIONS FOR COSMOLOGICAL RECOMBINATION}

\subsection{Hydrogen}

For the $1 S-2 P$ Ly $\alpha$ transition of hydrogen, $\lambda_{0}=1216 \AA$, $A_{21}=6.262 \times 10^{8} \mathrm{~s}^{-1}, f_{12}=0.4162$, and $\left(g_{2} / g_{1}\right)=3$, yielding an exceedingly large Sobolev optical depth, $\tau_{\mathrm{H}}=7.5 \times$ $10^{8} X_{\mathrm{H}}\left[(1+z) / 10^{3}\right]^{3 / 2}$, where $X_{\mathrm{H}}$ denotes the neutral fraction of hydrogen. The thermally broadened Ly $\alpha$ line has an effective cross section of $\langle\sigma\rangle \sim 3 \times 10^{-17} \mathrm{~cm}^{2}$ (Peebles 1993, p. 168) and a corresponding mean free path of $1 / n_{\mathrm{H}}\langle\sigma\rangle \sim 10^{14} \mathrm{~cm}$, at $z \sim 10^{3}$. Only on smaller scales can Ly $\alpha$ viscosity damp velocity gradients effectively. On the much larger scales in which the $\mathrm{CMB}$ anisotropies are measurable, the drag time in equation (7) is lengthened by a factor of $\sim \tau_{\mathrm{H}}$, which makes it very much longer than the Hubble time. The situation is similar for deuterium. Balmer line transitions of hydrogen have a small optical depth, but the occupation probability of their lower level is suppressed by an exceedingly large Boltzmann factor, $\sim \exp \left[-4.35 \times 10^{4} /(1+z)\right]$.

The resulting drag force is small, even when including the enhancement in the Ly $\alpha$ flux beyond the blackbody value, because of the $\operatorname{Ly} \alpha$ photons released during recombination $(\sim 1$ Ly $\alpha$ photon per hydrogen atom; see Dell'Antonio \& Rybicki 1993 for the distorted spectrum).

\subsection{Lithium}

\subsubsection{Drag Force and Opacity}

Lithium has a closed $n=1$ shell with two electrons and one electron outside this shell. The transition between the ground state $(2 S)$ and the first excited state $(2 P)$ has $\lambda_{0}=6708 \AA$ and $A_{21}=3.69 \times 10^{7} \mathrm{~s}^{-1}$, with $f_{12}=0.247$ and $\left(g_{2} / g_{1}\right)=1$ for the $2^{2} S-2^{2} P_{1 / 2}^{0}$ transition and $f_{12}=0.494$ and $\left(g_{2} / g_{1}\right)=2$ for the $2^{2} S-2^{2} P_{3 / 2}^{0}$ transition (Radzig \& Smirnov 1985, p. 224; Yan $\&$ Drake 1995). We therefore get, from equations (7) and (8),

$$
\begin{aligned}
H t_{\mathrm{Li}_{\mathrm{I}}}= & 4.96 \times 10^{-4}\left(\frac{1+z}{10^{3}}\right)^{5 / 2}\left(\frac{X_{\mathrm{Li}_{1}}}{3.8 \times 10^{-10}}\right)^{-1} \\
& \times \exp \left(\frac{7.89 \times 10^{3}}{1+z}\right)
\end{aligned}
$$

and

$$
\tau_{\mathrm{Li} \text { I }}=2.82 \frac{X_{\mathrm{Li} \text { I }}}{3.8 \times 10^{-10}}\left(\frac{1+z}{10^{3}}\right)^{3 / 2} .
$$

Figure 1 depicts the redshift dependence of the drag time in equation (9) and compares it with the drag time due to Thomson scattering in the recombining plasma,

$$
H t_{e}=\frac{3 m_{p} c H}{4 \sigma_{\mathrm{T}} a T^{4} X_{e}}=4.92 \times 10^{-3} X_{e}^{-1}\left(\frac{1+z}{10^{3}}\right)^{-5 / 2},
$$

where $X_{e}=\left(n_{e} / n_{p}\right)=n_{e} /\left(n_{e}+n_{\mathrm{H}}\right)$ is the ionization fraction of hydrogen. We calculated $X_{e}(z)$ from the updated version of the standard recombination model (Seager, Sasselov, \& Scott 2000). Despite the low abundance of lithium, its resonant drag force is not much lower than the Thomson drag force at $z \sim$ 500 , when roughly half of the lithium atoms are expected to have recombined (Palla, Galli, \& Silk 1995; Stancil, Lepp, \& Dalgarno 1996, 1998).

The net effect of the lithium drag is highly sensitive to the 


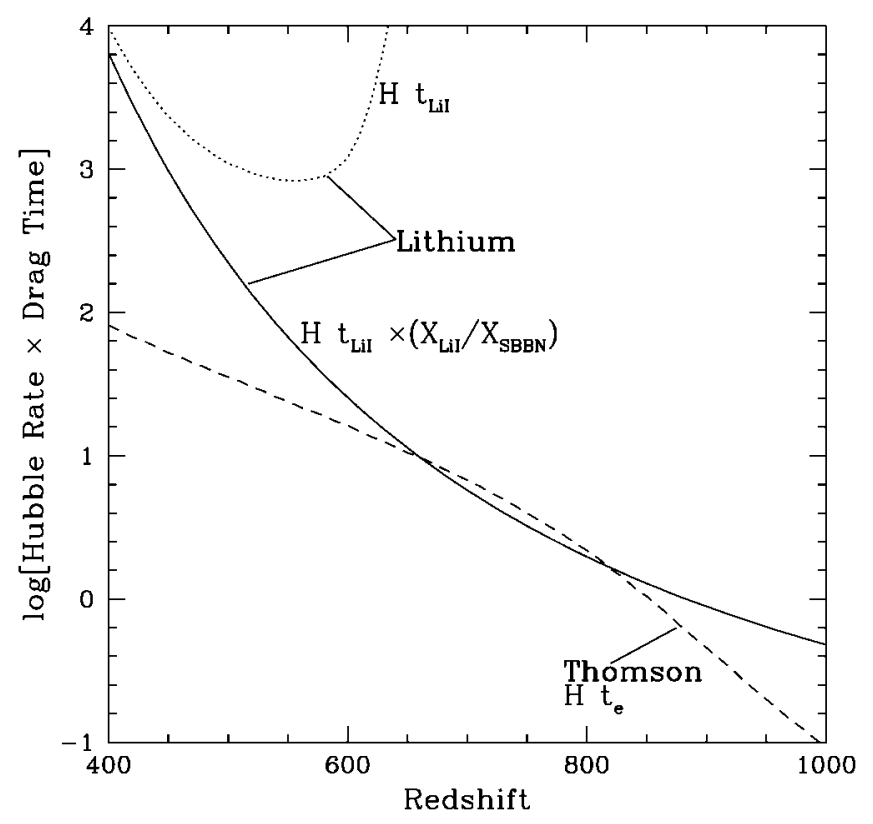

FIG. 1.-Redshift evolution of the dynamical drag time due to the Li $6708 \AA$ resonance with the microwave background. The drag time shown by the solid line should be divided by the neutral fraction of the primordial lithium abundance $\left(X_{\mathrm{SBBN}}=3.8 \times 10^{-10}\right.$; see Burles et al. 2001), as a function of redshift. The dotted line shows the result for one of the recombination histories of lithium in the literature (Palla et al. 1995). For comparison, the Thomson drag time is plotted as the dashed line, taking account of the full recombination history of hydrogen. In all cases, the drag times are normalized by the Hubble time, $H^{-1}(z)$.

recombination history of lithium and hydrogen. Previous discussions (e.g., Stancil et al. 1996, 1998) did not include the delaying effect of $\operatorname{Ly} \alpha$ photons on the recombination history of hydrogen (Peebles 1993) and therefore underestimated the free electron fraction and correspondingly the neutral lithium abundance at $z \lesssim 10^{3}$. Another process with an opposite sign that was omitted involves the excitation of neutral lithium from its ground level by the CMB radiation field. For this effect, it is important to take account of the Lyman and Balmer series distortions of the CMB spectrum (Dell'Antonio \& Rybicki 1993), which redshift into resonance at lithium recombination. In fact, the lithium chemistry in the early universe might provide a way for inferring the existence of these distortions. Finally, the recombination history of ${ }^{7} \mathrm{Be}$ should also be followed since a substantial fraction of the ${ }^{7} \mathrm{Li}$ forms through electron capture in the ${ }^{7} \mathrm{Be}$ produced by big bang nucleosynthesis (T. Walker 2001, private communication). Since the halflife of neutral ${ }^{7} \mathrm{Be}$ is only 53 days, this conversion can be regarded as instantaneous on the cosmological timescale, as soon as ${ }^{7} \mathrm{Be}$ recombines. Careful calculations of the neutral lithium fraction as a function of redshift are required in order to quantify the imprint of the lithium drag on the CMB anisotropies (A. Dalgarno, A. Loeb, \& P. Stancil 2001, in preparation).

The lithium chemistry could also be affected by a large drift velocity between the $\mathrm{Li}$ I fluid and the hydrogen plasma. To examine this effect, we consider the equation of motion for the Li I fluid (Burgers 1969),

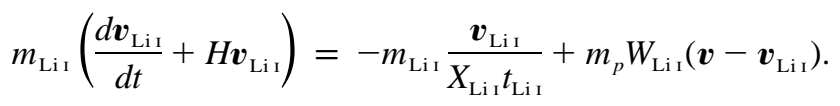

Here $W_{\mathrm{Li} \text { I }}=\Sigma_{k} \mu_{k: \mathrm{Li}} W_{k: \mathrm{Li}}$ is the collision rate of a $\mathrm{Li}$ atom with the gas, where $W_{k: \mathrm{LiI}}=n_{k}\left\langle\sigma_{k: \mathrm{Li} i} v\right\rangle$ and $\mu_{k: \mathrm{Li}}=m_{k} m_{\mathrm{Li}} /\left[m_{p} \times\right.$ $\left.\left(m_{k}+m_{\mathrm{Li}}\right)\right]$. The index $k$ runs over all other particle species in the gas that are assumed to move at a common bulk velocity $\boldsymbol{v}$ because they are not subject to the resonant drag force. The geometric cross section of $\mathrm{Li}$ I atoms, $\sigma_{\mathrm{Li} \text { I }} \sim 10^{-15} \mathrm{~cm}^{2}$, couples them to the rest of the gas on a timescale of $\sim 10^{7} \mathrm{~s}$, much shorter than the Hubble time, $H^{-1} \sim 3 \times 10^{13} \mathrm{~s}$ at $z \sim 10^{3}$. Hence, we may ignore the terms on the left-hand side of equation (12), as well as the perturbed gravitational force that is of the same order. The remaining terms give the solution

$$
\frac{\left(v-v_{\mathrm{LiI}}\right)}{v}=\frac{1}{1+Y},
$$

where $Y=W_{\mathrm{Li}} t_{\mathrm{Li} 1} X_{\mathrm{Li}} / A_{\mathrm{Li}}$. Substituting $A_{\mathrm{Li}}=7$, we find that $W_{\text {Li I }} \approx 10^{-7}\left[(1+z) / 10^{3}\right]^{3.5} \mathrm{~s}^{-1}$ and that $Y \gg 1$ at $z \lesssim 400$, after lithium recombination. At higher redshifts, the assumption that $v_{\mathrm{Li} 1}=v$ seems not to apply, and one may wonder whether the drag time in equation (6) should be multiplied by a factor of $\left(v / v_{\mathrm{Li} 1}\right)=(1+Y) / Y>1$ since the drag force acts on $\boldsymbol{v}_{\mathrm{Li} \text { I }}$ and not on $\boldsymbol{v}$. In this context, it is crucial to realize that the Li I nuclei do not maintain their identity as neutral atoms; in fact, they continuously form and get ionized on a timescale much shorter than a Hubble time. The cross section for Coulomb collisions of $\mathrm{Li}^{+}$is far greater than that of $\mathrm{Li} \mathrm{I}, \sigma_{\mathrm{Li}^{+}}=$ $2 \sqrt{\pi} e^{4} \ln \Lambda /(k T)^{2}=2.7 \times 10^{-11}\left[(1+z) / 10^{3}\right]^{-2} \mathrm{~cm}^{2}$, where $\ln \Lambda \approx 20$ is the Coulomb logarithm. The fraction of time spent by a $\mathrm{Li}$ nucleus in an ionized state is the ionization fraction, $f_{+}=X_{\mathrm{Li}^{+}} /\left(X_{\mathrm{Li}_{\mathrm{i}}}+X_{\mathrm{Li}^{+}}\right)$, and so $\sigma_{\mathrm{Li}}$ should be replaced by $\left[f_{+} \sigma_{\mathrm{Li}^{+}}+\left(1-f_{+}\right) \sigma_{\mathrm{Li}}\right]$ and the drag force term should be multiplied by $\left(1-f_{+}\right)$in equation (12). We consequently find that the substantial ionization fraction of the lithium fluid (Stancil et al. 1996, 1998) diminishes its drift velocity relative to the gas at all relevant redshifts, $z \leq 10^{3}$. Hence, nonthermal relative velocities may be ignored in the Li I chemistry.

At $z \lesssim 10^{3}$, the mean free path for resonant scattering by lithium exceeds the relevant spatial scales for the CMB anisotropies $\left(10^{23}-10^{24} \mathrm{~cm}\right)$, even if the lithium abundance is taken to be somewhat higher than the value, $X_{\mathrm{SBBN}}=3.8 \times 10^{-10}$, predicted by the latest deuterium measurements and standard big bang nucleosynthesis (Burles, Nollett, \& Turner 2001; see also Walker et al. 1991 and Smith, Kawano, \& Malaney 1993). Note that values as high as $X_{\mathrm{Li} \text { i }} \sim 10^{-8}$ were suggested by models of inhomogeneous big bang nucleosynthesis (Applegate \& Hogan 1985; Sale \& Mathews 1986; Mathews, Alcock, \& Fuller 1990) and would result in $H t_{\mathrm{Li} \text { I }} \lesssim 10$ for velocity gradients on sufficiently small scales with $\tau_{\mathrm{Li} \text { I }} \lesssim 1$.

\subsubsection{Effects on CMB Anisotropies}

If a substantial fraction of lithium recombines by $z \sim 500$ (as predicted by Palla et al. 1995 or Stancil et al. 1996, 1998), then the flux of the original anisotropies will be suppressed by the absorption factor, $\exp \left(-\tau_{\mathrm{Li} 1}\right)$, at observed wavelengths below $(6708 \AA \times 500)=335 \mu \mathrm{m}$, for which $\tau_{\mathrm{Li}} \approx 1.0\left(X_{\mathrm{Li}} / X_{\mathrm{SBBN}}\right)$. The resonant-scattering effect is different from that caused by Thomson scattering at the epoch of reionization (Hu \& White 1997) in that it has a larger optical depth and occurs within a much thinner shell of gas (for a sufficiently narrow band of observed wavelengths). Consequently, it should induce new firstorder anisotropies [multiplied by $\left.1-\exp \left(-\tau_{\mathrm{Li}}\right)\right]$ because of the coherence of the velocity field in the thin scattering shell. The dominant contribution to the new anisotropies would come 
from the Doppler effect. In contrast to reionization, there should be no severe cancellations of the Doppler effects from line-ofsight velocity fluctuations at large wavenumbers because the last scattering surface (or "visibility function") for the resonance is extremely thin. The increase in amplitude of subhorizon fluctuations between $z=10^{3}$ and $z=500$ would enhance the new anisotropies relative to the original ones and introduce a distortion of order unity in the original anisotropy spectrum on these scales. The anisotropy spectrum would be modified at multipole moments $l \gtrsim 100$, around and below the angular scale of the first acoustic peak (which is a factor of $\sim \sqrt{6}=2.4$ smaller than the horizon scale at $z \sim 500$ ), where the original anisotropy amplitude reaches its maximum $(l \approx 220)$. Resonant scattering would also result in enhanced polarization anisotropies on the same angular scales (Chandrasekhar 1960, p. 50). Since the scattering is done by lithium, the anisotropies will reflect the peculiar velocity of the lithium fluid that, as shown above, is not expected to deviate significantly from the velocity of the gas as a whole.

Since the temperature fluctuations are in the Wien tail of the $\mathrm{CMB}$ spectrum, they translate to brightness fluctuations (in units of ergs $\mathrm{s}^{-1} \mathrm{~cm}^{-2} \mathrm{sr}^{-1} \mathrm{~Hz}^{-1}$ ) of a much larger contrast,

$$
\frac{\Delta B_{v}}{B_{v}}=\frac{d \ln B_{v}}{d \ln T} \frac{\Delta T}{T}=\frac{h \nu}{k T} \frac{\Delta T}{T}=15.78 \frac{500}{1+z} \frac{\Delta T}{T},
$$

where $z$ is the redshift being probed, and we have substituted $B_{v}(T) \propto \exp (-h \nu / k T)$. Hence, the first acoustic peak would correspond to brightness fluctuations of up to $\sim 5 \times 10^{-4}$.

At a given observed wavelength, the redshift thickness of the scattering shell is only determined by two contributions: (1) the possibility that a photon at this wavelength was absorbed either by the $2^{2} S-2{ }^{2} P_{3 / 2}^{0}$ transition with $\lambda_{0}=$ $6707.76 \AA$ or at a slightly lower redshift by the $2^{2} S-2{ }^{2} P_{1 / 2}^{0}$ transition with $\lambda_{0}=6707.91 \AA$ (Radzig \& Smirnov 1985), yielding $[\Delta z /(1+z)]=2.2 \times 10^{-5}$, and $(2)$ the thermal width of the line, ${ }^{3}[\Delta z /(1+z)]=(\Delta \lambda / \lambda)_{\mathrm{th}} \approx 10^{-5}$. In practice, the minimum thickness would be limited by the bandwidth of the detector. The maximum bandwidth and minimum angular resolution should be tuned to match the expected coherence length of the peculiar velocity field at $z \sim 500$, which corresponds to $(\Delta \lambda / \lambda) \sim 0.2$ and $\Delta \theta \sim 6^{\prime}$ or, equivalently, $l \sim$ $10^{3}$.

Observations at different far-infrared (FIR) wavelengths would probe different thin slices of structure in the early universe. The anisotropy pattern would vary gradually as a function of wavelength, and "three-dimensional tomography" is in principle possible, by which one may probe correlations in the structure along the line of sight. The feasibility of this measurement might be severely compromised by the anisotropies of the FIR foreground. Unfortunately, $350 \mu \mathrm{m}$ is just the wavelength where the CMB intensity blends into the extragalactic FIR foreground (see Fig. 1 in Scott et al. 2001). However, the contribution of discrete sources (see Fig. 6 in Knox et al. 2001)

\footnotetext{
${ }^{3}$ Since $\tau_{\mathrm{Li} 1} \leqslant 1$, the photon does not need to redshift away from the line center by more than the line width in order to escape to infinity.

Applegate, J. H., \& Hogan, C. J. 1985, Phys. Rev. D, 31, 3037

Burgers, J. M. 1969, Flow Equations For Composite Gases (New York: Academic)

Burles, S., Nollett, K. M., \& Turner, M. S. 2001, ApJ, 552, L1
}

could be separated out through observations at shorter wavelengths with a higher angular resolution. Furthermore, continuum foreground emission would result in similar anisotropies for all wavelengths within a bandwidth $(\Delta \lambda / \lambda) \sim 1$, while the CMB anisotropies would vary considerably across such a band since its width corresponds to $\Delta z /(1+z) \sim 1$. Subtraction of anisotropy maps at slightly different wavelengths within such a band can therefore be used to isolate out the CMB component (as long as these wavelengths do not overlap with strong emission lines from the foreground sources). For $l \lesssim 100$, the anisotropies should have the same power spectrum as measured by MAP or Planck at much longer wavelengths. The predictable correlations between the temperature and polarization patterns of the CMB and their general statistical properties could be used to further enhance the signal-to-noise ratio (M. Zaldarriaga \& A. Loeb 2001, in preparation). Obviously, these difficult observations need to be made through holes in the Galactic dust emission at high Galactic latitudes.

\section{CONCLUSIONS}

The $6708 \AA$ resonant transition of neutral lithium provides a previously unexplored coupling between the baryons and the CMB after cosmological recombination. The lithium opacity is substantial at observed wavelengths $\lesssim 335\left[\left(1+z_{\mathrm{Li}}\right) / 500\right] \mu \mathrm{m}$, where $z_{\mathrm{Li}}$ is the redshift at which $\sim 50 \%$ of the lithium recombines. At these wavelengths, the CMB anisotropies on subdegree angular scales would be significantly altered because of the finite optical depth for resonant scattering, $\tau_{\mathrm{Li}_{\mathrm{I}}} \approx 0.5[(1+$ $\left.\left.z_{\text {Li }}\right) / 500\right]^{3 / 2}$. This scattering would generate new temperature and polarization anisotropies at multipole moments $l \gtrsim 100$. The detection of these anisotropies depends on the prospects for cleaning the expected anisotropies of the FIR foreground (Knox et al. 2001) and may be feasible through the subtraction of anisotropy maps for pairs of slightly different wavelengths.

The relevant wavelength range overlaps with the highest frequency channel of the Planck mission $(352 \mu \mathrm{m})$ and with the proposed balloon-borne Explorer of Diffuse Galactic Emissions ${ }^{4}$ experiment, which will survey $1 \%$ of the sky in 10 wavelength bands between 230 and $2000 \mu \mathrm{m}$ with a resolution ranging from $6^{\prime}$ to $14^{\prime}$ (see Table 1 in Knox et al. 2001). However, a new instrument design with multiple narrow bands $(\Delta \lambda / \lambda \lesssim 0.1)$ at various wavelengths in the range of $\lambda=$ $250-350 \mu \mathrm{m}$ and with an angular resolution of $\sim 6^{\prime}$ is necessary in order to optimize the detection of the lithium signature on the CMB anisotropies. The effects discussed in this Letter depend critically on the recombination history of lithium and emphasize the need to explore the related lithium chemistry in more detail in the future.

The author thanks Alex Dalgarno, Daniel Eisenstein, George Rybicki, and Matias Zaldarriaga for useful discussions. This work was supported in part by NASA grants NAG5-7039 and NAG5-7768 and by NSF grants AST 99-00877 and AST 0071019.

${ }^{4}$ See http://topweb.gsfc.nasa.gov.

\section{REFERENCES}

Chandrasekhar, S. 1960, Radiative Transfer (New York: Dover)

Dell'Antonio, I. P., \& Rybicki, G. B. 1993, in ASP Conf. Ser. 51, Observational Cosmology, ed. G. Chincarini, A. Iovino, T. Maccacaro, \& D. Maccagni (San Francisco: ASP), 548 
Hu, W., \& White, M. 1997, ApJ, 479, 568

Knox, L., Cooray, A., Eisenstein, D., \& Haiman, Z. 2001, ApJ, 550, 7

Mather, J. C., Fixsen, D. J., Shafer, R. A., Mosier, C., \& Wilkinson, D. T. 1999, ApJ, 512, 511

Mathews, G. J., Alcock, C. R., \& Fuller, G. M. 1990, ApJ, 349, 449

Palla, F., Galli, D., \& Silk, J. 1995, ApJ, 451, 44

Peebles, P. J. E. 1993, Principles of Physical Cosmology (Princeton: Princeton Univ. Press)

Peebles, P. J. E., \& Yu, J. T. 1970, ApJ, 162, 815

Radzig, A. A., \& Smirnov, B. M. 1985, Reference Data on Atoms, Molecules, and Ions (New York: Springer)

Rybicki, G. B., \& Hummer, D. G. 1978, ApJ, 219, 654

Rybicki, G. B., \& Lightman, A. P. 1979, Radiative Processes in Astrophysics (New York: Wiley)

Sale, K. E., \& Mathews, G. J. 1986, ApJ, 309, L1

Seager, S., Sasselov, D. D., \& Scott, D. 2000, ApJS, 128, 407
Scott, D. 2000, in Beyond the Desert 99: Second Int. Conf. on Particle Physics beyond the Standard Model, ed. H. V. Klapdor-Kleingrothaus \& I. V. Kvivosheina (Bristol: IOP)

Scott, D., Borys, C., Halpern, M., Sajina, A., Chapman, S., \& Fahlman, G. 2001, in UMass/INAOE Conf. Proc. on Deep Millimeter Surveys, ed. J. Lowenthal \& D. Hughes, in press (astro-ph/0010337)

Smith, M. S., Kawano, L. H., \& Malaney, R. A. 1993, ApJS, 85, 219

Sobolev, V. V. 1946, Moving Atmospheres of Stars (Leningrad: Leningrad State Univ.; English transl. 1960 [Cambridge: Harvard Univ. Press])

Stancil, P. C., Lepp, S., \& Dalgarno, A. 1996, ApJ, 458, 401 1998, ApJ, 509, 1

Yan, Z., \& Drake, G. W. F. 1995, Phys. Rev. A, 52, 4316

Yu, Q., Spergel, D. N., \& Ostriker, J. P. 2001, ApJ, in press (astro-ph/0103149) Walker, T. P., Steigman, G., Kang, H., Schramm, D. M., \& Olive, K. A. 1991, ApJ, 376, 51

White, M., Scott, D., \& Silk, J. 1994, ARA\&A, 32, 319 